\title{
An Analytical Approach to V-BLAST Detection with Optimal Ordering for Two Input Multiple Output Systems
}

\author{
Wei Peng ${ }^{1}$, Shaodan $\mathrm{Ma}^{2}$, Tung-Sang $\mathrm{Ng}^{2}$, Jiangzhou Wang ${ }^{3}$ and Fumiyuki Adachi ${ }^{1}$ \\ ${ }^{1}$ Department of Electrical and Communication Engineering, Tohoku University, Japan \\ ${ }^{2}$ Department of Electrical and Electronic Engineering, the University of Hong Kong, Hong Kong \\ ${ }^{3}$ Department of Electronics, the University of Kent, UK
}

\begin{abstract}
In this paper, an analytical approach to performance analysis of vertical Bell laboratories layered space time (V-BLAST) detection with optimal ordering for systems with two transmit antennas is presented. The post-detection signal-to-noise-ratio (SNR) at each stage is derived and the symbol error probabilities (SEP) of the signals are then given in closed-form. The analysis takes into account the effects of optimal ordering, imperfect channel estimation and error propagation, which were rarely considered in the literature due to the difficulties in evaluation. The accuracy of the analysis is demonstrated by Monte-Carlo simulations.

Key words: Symbol Error Probability, V-BLAST, Optimal Ordering, Imperfect Channel Estimation, Error Propagation
\end{abstract}

\section{INTRODUCTION}

The vertical Bell laboratories layered space time (V-BLAST) detection algorithm [1] is the first and still popular non-linear detection algorithm for multiple-input multiple-output (MIMO) receivers, due to its low implementation complexity. A number of studies on its performance analysis have been reported in [2 - 6]. The solutions found are limited in some way, since they are generally derived based on assumptions that decisions in previous stages are correct $[2,3]$, the channel is perfectly estimated [2 - 5] and/or no ordering is adopted [4], while the work in [6] uses an assumption that an error in one stage will cause an error in its following stage with probability one. However, these assumptions usually do not fit into the real situation. Furthermore, in order to mitigate the effect of error propagation, optimal ordering based on the post-detection signal-to-noise-ratio (SNR) is generally adopted. The presence of errors and the adoption of the optimal ordering would significantly affect the performance of V-BLAST detection. It is thus of great interest to analyze the performance of V-BLAST detection simultaneously taking into account the error propagation, channel estimation errors as well as the optimal ordering. Such analyses, especially optimal ordering, were rarely considered in the literature.

In this paper, an analytical approach is presented to evaluate the symbol error probability (SEP) of V-BLAST detection with optimal ordering. The simplest system with two transmit antennas are considered. By modeling the channel estimation errors and the decision error as equivalent noises, the post-detection SNR at each detection stage is developed and the SEPs of the two transmitted signals after optimal ordering are then sequentially derived in closed-form. The accuracy of this analytical approach is finally demonstrated by Monte Carlo simulations.

\section{SYSTEM MODEL AND V-BLAST DETECTION}

\section{A. System model with imperfect channel estimation}

For a MIMO system with two transmit and $N_{r}$ receive antennas $\left(N_{r} \geq 2\right)$, the base-band received signal vector is given by

$$
\mathbf{y}=\mathbf{H x}+\mathbf{n},
$$

where $\mathbf{y}=\left[y_{1}, \cdots, y_{N_{r}}\right]^{T}$ and $\mathbf{x}=\left[x_{1}, x_{2}\right]^{T}$ are the received signal vector and the transmitted signal vector, respectively; superscript $T$ represents matrix transpose; $\mathbf{H}$ is an $N_{r} \times 2$ channel matrix, whose elements are independently and identically distributed (i.i.d.) complex Gaussian random variables with zero mean and unit variance $\left(\sigma_{h}^{2}=1\right) ; \mathbf{n}=\left[n_{1}, \cdots, n_{N_{r}}\right]^{T}$ is the noise vector with each element being i.i.d. complex Gaussian variable with zero mean and variance $\sigma_{n}^{2}$. Let $C$ represent the constellation of the transmitted signals. It is assumed that all the constellation symbols have equal probability. To simplify the derivation, quadrature phase shift keying (QPSK) modulation is assumed here and this analysis can be easily extended to the system using other modulation schemes.

Denoting $\hat{\mathbf{H}}$ to be the estimated channel matrix, the elements of $\hat{\mathbf{H}}$ are i.i.d. complex Gaussian variables with zero mean and variance $\sigma_{\hat{h}}^{2}$. $\mathbf{H}$ can be written as [7]

$$
\mathbf{H}=\rho_{h, \hat{h}} \hat{\mathbf{H}}+\Delta \mathbf{H},
$$

where $\rho_{h, \hat{h}}=c_{h, \hat{h}} / \sigma_{\hat{h}}, c_{h \hat{h}}$ is the correlation coefficient between the corresponding elements of $\hat{\mathbf{H}}$ and $\mathbf{H}, 0<c_{h, \hat{h}} \leq 1$; $\Delta \mathbf{H}$ represents the channel estimation error matrix and its elements are i.i.d. complex Gaussian variables with zero mean and variance $\sigma_{\Delta h}^{2}=1-c_{h, \hat{h}}^{2}$. Under any given SNR, $\rho_{h, \hat{h}}$ and $c_{h \hat{h}}$ are assumed to be known at the receiver [8].

$h$ Substituting (2) into (1) yields

$$
\mathbf{y}=\left(\rho_{h, \hat{h}} \hat{\mathbf{H}}+\Delta \mathbf{H}\right) \mathbf{x}+\mathbf{n}=\rho_{h, \hat{h}} \hat{\mathbf{H}} \mathbf{x}+\mathbf{u},
$$

where $\mathbf{u}=\Delta \mathbf{H x}+\mathbf{n}$. It is clear that given $\mathbf{x}$, the elements of $\mathbf{u}$ are complex Gaussian variables [9] with zero mean and variance $\sigma_{u}^{2}=E_{s}\left(1-c_{h, \hat{h}}^{2}\right)+\sigma_{n}^{2}$, where $E_{s}=\sum_{i=1}^{2}\left|x_{i}\right|^{2}$ denotes the total

\section{B. V-BLAST detection with optimal ordering}

It is shown in [10] that the optimal ordering rule is to detect the transmitted signals according to the energy at the receiver in decreasing order. Here we adopt this ordering rule and the detection order is accordingly determined by the norm of each column of the estimated channel matrix. Let $\hat{\mathbf{h}}_{i}$ represent the $i^{\text {th }}$ column of $\hat{\mathbf{H}}$ and $\left\|\hat{\mathbf{h}}_{i}\right\|$ represent the norm of $\hat{\mathbf{h}}_{i}$. Assume that $\left\|\hat{\mathbf{h}}_{r(1)}\right\| \leq\left\|\hat{\mathbf{h}}_{r(2)}\right\|$, where $r(2)$ is the index of the column vector 
with larger norm while $r(1)$ is the index of the column vector with smaller norm. Following the optimal ordering, the detection should be performed first on signal $x_{r(2)}$ and then on signal $x_{r(1)}$.

$\mathrm{V}$-BLAST detection can be realized with the aid of QR decomposition. Reordering the transmit signals as $\overline{\mathbf{x}}=\left[\begin{array}{ll}x_{r(1)} & x_{r(2)}\end{array}\right]^{T}$, the received signal vector in (3) is rewritten as

$$
\begin{aligned}
\mathbf{y} & =\rho_{h, \hat{h}}\left[\begin{array}{lll}
\hat{h}_{1, r(1)} & \cdots & \hat{h}_{N_{r}, r(1)} \\
\hat{h}_{1, r(2)} & \cdots & \hat{h}_{N_{r}, r(2)}^{T}
\end{array}\right]^{T}\left[\begin{array}{c}
x_{r(1)} \\
x_{r(2)}
\end{array}\right]+\mathbf{u}, \\
& =\rho_{h, h} \overline{\mathbf{H}} \overline{\mathbf{x}}+\mathbf{u}
\end{aligned}
$$

where $\overline{\mathbf{H}}=\left[\begin{array}{lll}\hat{h}_{1, r(1)} & \cdots & \hat{h}_{N_{r}, r(1)} \\ \hat{h}_{1, r(2)} & \cdots & \hat{h}_{N_{r}, r(2)}\end{array}\right]^{T}$. Let $\overline{\mathbf{H}}=\overline{\mathbf{Q}} \overline{\mathbf{R}}$ where $\overline{\mathbf{Q}}$ is a unitary matrix and $\overline{\mathbf{R}}$ is an upper triangular matrix. For the purpose of detection, a signal vector can be formed as

$$
\overline{\mathbf{z}}=\overline{\mathbf{Q}}^{H} \mathbf{y}=\overline{\mathbf{Q}}^{H}\left(\rho_{h, h} \overline{\mathbf{H}} \overline{\mathbf{x}}+\mathbf{u}\right)=\rho_{h, h} \overline{\mathbf{R}} \overline{\mathbf{x}}+\overline{\mathbf{g}},
$$

where $\overline{\mathbf{g}}=\overline{\mathbf{Q}}^{H} \mathbf{u}$ is the noise vector after the matrix multiplication. Apparently, each element of $\overline{\mathbf{g}}$ is independent complex Gaussian variable with zero mean and variance $\sigma_{g}^{2}=\sigma_{u}^{2}[11]$ because that the unitary transform will not change the statistic of the noise vector $\mathbf{u}$. By expanding (5), we can get

$$
\begin{array}{r}
\rho_{h, \hat{h}} \bar{r}_{1,1} x_{r(1)}+\rho_{h, \hat{h}} \bar{r}_{1,2} x_{r(2)}+\bar{g}_{1}=\bar{z}_{1} \\
\rho_{h, \hat{h}} \bar{r}_{2,2} x_{r(2)}+\bar{g}_{2}=\bar{z}_{2},
\end{array}
$$

where $\bar{r}_{j, i}$ is the $(j, i)^{\text {th }}$ element of $\overline{\mathbf{R}}, \bar{z}_{j}$ and $\bar{g}_{j}$ are respectively the $j^{\text {th }}$ element of $\overline{\mathbf{z}}$ and $\overline{\mathbf{g}}$. Given (6), V-BLAST detection will be carried out sequentially in two stages as follows. At the $1^{\text {st }}$ stage, signal $x_{r(2)}$ is detected as

$$
\tilde{x}_{r(2)}=\operatorname{slice}\left(\hat{x}_{r(2)}\right) \text {, }
$$

where $\hat{x}_{r(2)}=\bar{z}_{2} / \rho_{h, \hat{h}} \bar{r}_{2,2}$ and $\tilde{x}_{r(2)}$ stands for the decision of $x_{r(2)}$; symbol stice $(\cdot)$ represents the operation to map $\hat{x}_{r(2)}$ to the nearest symbol in the constellation. Then at the $2^{\text {nd }}$ stage, the interference from $x_{r(2)}$ is subtracted from $\bar{z}_{1}$ using the decision $\tilde{x}_{r(2)}$ and the signal $x_{r(1)}$ will be detected as

$$
\tilde{x}_{r(1)}=\operatorname{slice}\left(\hat{x}_{r(1)}\right) \text {, }
$$

where $\hat{x}_{r(1)}=\left(\bar{z}_{1}-\rho_{h, \hat{h}} \bar{r}_{1,2} \tilde{x}_{r(2)}\right) / \rho_{h, h} \bar{r}_{1,1}$.

\section{Statistics of the upper triangular matrix $\overline{\mathbf{R}}$}

The statistics of the elements in the upper triangular matrix $\overline{\mathbf{R}}$ is essential to the SEP analysis. Previous studies have evaluated the statistics in $[3,12]$. Useful results for the analysis are summarized as follows. 1) When no ordering is performed, the $(j, i)^{t h}(j<i)$ element of $\overline{\mathbf{R}}$ is complex Gaussian variable with zero mean and variance $\sigma_{\hat{h}}^{2}$; while the squares of the normalized diagonal elements, defined as $\omega_{i}=\bar{r}_{i, i}^{2} / \sigma_{\hat{h}}^{2},(i=1,2)$, follow the central chi-square distribution with the degrees of freedom equals to $2\left(N_{r}-i+1\right)$. 2) The optimal ordering will mainly change the statistics of the diagonal elements. The probability density function (p.d.f) of $\omega_{1}$ and $\omega_{2}$ after optimal ordering are respectively given by [3]

$$
p\left(\omega_{1}\right)=\frac{2 \omega_{1}^{N_{r}-1} \exp \left(-2 \omega_{1}\right)}{\left(N_{r}-1\right) !}\left(\sum_{k=0}^{N_{r}-1} \frac{1}{k !} \omega_{1}^{k}\right)
$$

and

$$
\begin{aligned}
& p\left(\omega_{2}\right) \\
& =\frac{2 \omega_{2}^{N_{r}-2}}{\left(N_{r}-1\right) !}\left[\begin{array}{l}
\exp \left(-\omega_{2}\right) \\
\left.-\exp \left(-2 \omega_{2}\right) \sum_{k=0}^{N_{r}-1} \frac{1}{2^{k+1}} \sum_{m=0}^{k} \frac{1}{m !} 2^{m} \omega_{2}^{m}\right]
\end{array}\right.
\end{aligned}
$$

\section{SEP ANALYSIS}

A. SEP of signal $x_{r(2)}$ in the $1^{\text {st }}$ stage: $P\left(\tilde{x}_{r(2)} \neq x_{r(2)}\right)$

At the $1^{\text {st }}$ detection stage, signal $x_{r(2)}$ is detected as $\tilde{x}_{r(2)}=\operatorname{slice}\left(\hat{x}_{r(2)}\right)$. The post-detection SNR can be calculated by

$$
\gamma_{2}=\rho_{h, \hat{h}}^{2} \bar{r}_{2,2}^{2}\left|x_{r(2)}\right|^{2} / \sigma_{g}^{2}=c_{h, h}^{2} \omega_{2}\left|x_{r(2)}\right|^{2} / \sigma_{u}^{2} .
$$

It follows that given $x_{r(2)}$ and $\omega_{2}$, the SEP for QPSK is written as [9]

$$
\begin{aligned}
& p\left(\tilde{x}_{r(2)} \neq x_{r(2)} \mid x_{r(2)}, \omega_{2}\right)=G\left(\gamma_{2}\right) \\
& =2 Q\left(\sqrt{\gamma_{2}}\right)-Q^{2}\left(\sqrt{\gamma_{2}}\right) \\
& =\frac{1}{\pi} \int_{0}^{3 \pi / 4} \exp \left(-c_{h, h}^{2} \omega_{2}\left|x_{r(2)}\right|^{2} / 2 \sigma_{u}^{2} \sin ^{2} \theta\right) d \theta
\end{aligned}
$$

where $\quad Q(A)=\int_{1}^{\infty} 1 / \sqrt{2 \pi} \exp \left(-t^{2} / 2\right) d t$

Equation $2 Q(\sqrt{A})-Q^{2}(\sqrt{A})=1 / \pi \cdot \int_{0}^{3 \pi / 4} \exp \left(-A / 2 \sin ^{2} \theta\right) d \theta[13]$ is used in (12). It should be noted that the function $G(\cdot)$ depends on the modulation scheme. It is straight forward to apply this method to the system using other modulation schemes by altering $G(\cdot)$. By averaging (12) with respect to the statistics of $x_{r(2)}$ and $\omega_{2}$, the average SEP can be numerically evaluated as

$$
\begin{aligned}
& P\left(\tilde{x}_{r(2)} \neq x_{r(2)}\right) \\
& =\sum_{x_{r(2)} \in C} p\left(x_{r(2)}\right) \int_{0}^{\infty} p\left(\tilde{x}_{r(2)} \neq x_{r(2)} \mid x_{r(2)}, \omega_{2}\right) p\left(\omega_{2}\right) d \omega_{2} .
\end{aligned}
$$

Applying (10) and (12) into (13), the SEP of the signal in the $1^{\text {st }}$ stage will be obtained.

\section{B. SEP of the signal $x_{r(1)}$ in the $2^{\text {nd }}$ stage: $P\left(\tilde{x}_{r(1)} \neq x_{r(1)}\right)$}

At the $2^{\text {nd }}$ detection stage, the SEP $P\left(\tilde{x}_{r(1)} \neq x_{r(1)}\right)$ can be expressed as

$$
\begin{aligned}
& P\left(\tilde{x}_{r(1)} \neq x_{r(1)}\right) \\
& =P\left(\tilde{x}_{r(1)} \neq x_{r(1)} \mid \tilde{x}_{r(2)}=x_{r(2)}\right)\left[1-P\left(\tilde{x}_{r(2)} \neq x_{r(2)}\right)\right], \\
& +P\left(\tilde{x}_{r(1)} \neq x_{r(1)} \mid \tilde{x}_{r(2)} \neq x_{r(2)}\right) P\left(\tilde{x}_{r(2)} \neq x_{r(2)}\right)
\end{aligned}
$$


where $\left.P\left(\tilde{x}_{r(1)} \neq x_{r(1)}\right) \tilde{x}_{r(2)}=x_{r(2)}\right)$ and $P\left(\tilde{x}_{r(1)} \neq x_{r(1)} \tilde{x}_{r^{\prime(2)}} \neq x_{r(2)}\right)$ represent the SEP of $x_{r(1)}$ under the condition of $\tilde{x}_{r(2)}=x_{r(2)}$ and $\tilde{x}_{r(2)} \neq x_{r(2)}$ respectively. Here the error propagation is effectively modeled by introducing conditional SEPs. Next, the conditional SEPs will be derived.

B.1 The conditional SEP $P\left(\tilde{x}_{r(1)} \neq x_{r(1)} \mid \tilde{x}_{r(2)}=x_{r(2)}\right)$

Recall $\tilde{x}_{r(1)}=$ slice $\left(\hat{x}_{r(1)}\right)$ in (8), where $\hat{x}_{r(1)}=\left(\bar{z}_{1}-\rho_{\left.h, \hat{h}_{1,2} \bar{x}_{r(2)}\right) / \rho_{h, h} \overline{\bar{r}}_{1,1}}\right.$. Under the condition of $\tilde{x}_{r(2)}=x_{r(2)}$, it follows from (6) that

$$
\tilde{x}_{r(1)}=\operatorname{slice}\left(\frac{\rho_{h, \hat{h}} \bar{h}_{1,1} x_{r(1)}+g_{1}}{\rho_{h, \hat{h}} \bar{r}_{1,1}}\right) .
$$

Given (15), the post-detection SNR can be evaluated as

$$
\gamma_{1, \tilde{x}_{(2)}=x_{r(2)}}=\rho_{h, \hat{h}}^{2} \bar{h}_{1,1}^{2}\left|x_{r(1)}\right|^{2} / \sigma_{g}^{2}=c_{h, \hat{h}}^{2} \omega_{1}\left|x_{r(1)}\right|^{2} / \sigma_{u}^{2} .
$$

It follows that the SEP conditioned on $x_{r(1)}, \omega_{1}$ and $\tilde{x}_{r(2)}=x_{r(2)}$ is given as

$$
\begin{aligned}
& p\left(\tilde{x}_{r(1)} \neq x_{r(1)} \mid x_{r(1)}, \bar{\omega}_{1}, \tilde{x}_{r(2)}=x_{r(2)}\right)=G\left(\gamma_{1, \tilde{r}_{r(2)}=x_{r(2)}}\right) . \\
& =\frac{1}{\pi} \int_{0}^{3 \pi / 4} \exp \left(-c_{h, \hat{h}}^{2} \bar{\omega}_{1}\left|x_{r(1)}\right|^{2} / 2 \sigma_{u}^{2} \sin ^{2} \theta\right) d \theta
\end{aligned} .
$$

By averaging (17) with respect to the statistics of $x_{r(1)}$ and $\omega_{1}$, the average conditional SEP $P\left(\tilde{x}_{r(1)} \neq x_{r(1)} \mid \tilde{x}_{r(2)}=x_{r(2)}\right)$ is then obtained using (9) and (17) as

$$
\begin{aligned}
& P\left(\tilde{x}_{r(1)} \neq x_{r(1)} \mid \tilde{x}_{r(2)}=x_{r(2)}\right) \\
= & \sum_{x_{r(1)} \in C} p\left(x_{r(1)}\right) \int_{0}^{\infty} p\left(\tilde{x}_{r(1)} \neq x_{r(1)} \mid x_{r(1)}, \omega_{1}, \tilde{x}_{r(2)}=x_{r(2)}\right) p\left(\omega_{1}\right) d \omega_{1} .
\end{aligned}
$$

\section{B.2 The conditional SEP $P\left(\tilde{x}_{r(1)} \neq x_{r(1)} \mid \tilde{x}_{r(2)} \neq x_{r(2)}\right)$}

According to (6) and (8), under the condition $\tilde{x}_{r(2)} \neq x_{r(2)}$, the decision for $x_{r(1)}$ is made as

$$
\begin{aligned}
\tilde{x}_{r(1)} & =\operatorname{slice}\left(\frac{\rho_{h, \hat{h}} \overline{\bar{l}}_{1,1} x_{r(1)}+\rho_{h, \hat{h}} \overline{\bar{r}}_{1,2} \Delta x_{r(2)}+\bar{g}_{1}}{\rho_{h, \hat{h}} \bar{r}_{1,1}}\right), \\
& =\operatorname{slice}\left(\frac{\rho_{h, \hat{h}} \overline{\bar{h}}_{1,1} x_{r(1)}+v_{1}}{\rho_{h, \hat{h}} \bar{r}_{1,1}}\right)
\end{aligned}
$$

where $\Delta x_{r(2)}=x_{r(2)}-\tilde{x}_{r(2)}$ and $v_{1}=\rho_{h, h} \bar{r}_{1,2} \Delta x_{r(2)}+\bar{g}_{1}$. It follows that given $\Delta x_{r(2)}, v_{1}$ is a complex Gaussian variable with zero mean and variance $\sigma_{v}^{2}=c_{h, \hat{h}}^{2}\left|\Delta x_{r(2)}\right|^{2}+\sigma_{u}^{2}$. Since the error occurs in adjacent points in the constellation with highest probability, $\left|\Delta x_{r(2)}\right|^{2}$ can be approximated as $\left|\Delta x_{r(2)}\right|^{2} \approx \min \left(d_{r(2) t c}^{2}\right)=\alpha E\left\{\left|x_{r(2)}\right|^{2}\right\}$ where $d_{r(2), c}^{2}$ represents the minimum square Euclidean distance (SED) between $x_{r(2)}$ and its constellation neighbors and $E\left\{\left|x_{r(2)}\right|^{2}\right\}$ stands for the average symbol energy of $x_{r(2)}$. $\alpha$ varies with the modulation scheme. For QPSK modulation, $\alpha=2$.
From (19), the post-detection SNR under the condition of $\tilde{x}_{r(2)} \neq x_{r(2)}$ becomes

$$
\gamma_{1, \tilde{x}_{r(2)} \neq x_{r(2)}}=\rho_{h, \hat{h}}^{2} \bar{r}_{1,1}^{2}\left|x_{r(1)}\right|^{2} / \sigma_{v}^{2}=c_{h, \hat{h}}^{2} \omega_{1}\left|x_{r(1)}\right|^{2} / \sigma_{v}^{2} .
$$

Accordingly, the SEP conditioned on $x_{r(1)}, \omega_{1}$ and $\tilde{x}_{r(2)} \neq x_{r(2)}$ is expressed as

$$
\begin{aligned}
& p\left(\tilde{x}_{r(1)} \neq x_{r(1)} \mid x_{r(1)}, \omega_{1}, \tilde{x}_{r(2)} \neq x_{r(2)}\right)=G\left(\gamma_{1, \tilde{x}_{r(2)} \neq x_{r(2)}}\right) \\
& =\frac{1}{\pi} \int_{0}^{3 \pi / 4} \exp \left(-c_{h, \hat{h}}^{2} \omega_{1}\left|x_{r(1)}\right|^{2} / 2 \sigma_{v}^{2} \sin ^{2} \theta\right) d \theta
\end{aligned}
$$

It follows from (9) and (21) that the average conditional SEP

$$
\begin{aligned}
& P\left(\tilde{x}_{r(1)} \neq x_{r(1)} \mid \tilde{x}_{r(2)} \neq x_{r(2)}\right) \text { is equal to } \\
& \quad P\left(\tilde{x}_{r(1)} \neq x_{r(1)} \mid \tilde{x}_{r(2)} \neq x_{r(2)}\right) \\
& \quad=\sum_{x_{r(1)} \in C} p\left(x_{r(1)}\right) \int_{0}^{\infty} p\left(\tilde{x}_{r(1)} \neq x_{r(1)} \mid x_{r(1)}, \omega_{1}, \tilde{x}_{r(2)} \neq x_{r(2)}\right) p\left(\omega_{1}\right) d \omega_{1} .
\end{aligned}
$$

Now, the SEP $P\left(\tilde{x}_{r(1)} \neq x_{r(1)}\right)$ can be obtained by substituting (13), (18) and (22) into (14).

\section{NUMERICAL AND SIMULATION RESULTS}

A system with two transmit antennas and four receive antennas $\left(N_{r}=4\right)$ is taken as an example. Unit power is allocated to both transmit antennas $\left(E\left\{\left|x_{1}\right|^{2}\right\}=E\left\{\left|x_{2}\right|^{2}\right\}=1\right)$. The channel gains are randomly generated complex Gaussian variables with zero mean and unit variance. Perfect channel state information (CSI) and imperfect CSI at the receiver are assumed respectively. To study the effects of ordering, the results for the V-BLAST detection without ordering will also be shown for comparison (the performance analysis for V-BLAST detection without ordering is carried out but not presented for brevity). Since there is no direct mapping from the stage index to the transmit antennas when optimal ordering is adopted, the overall SEP performance (an average performance of the transmitted signals) is also meaningful. In the following, the SEP results will be given in terms of the average transmit power to the average noise power ratio $\left(S N R=1 / \sigma_{n}^{2}\right)$. The simulation results are obtained by averaging over $10^{6}$ Monte Carlo realizations.

\section{A. Comparisons between the analytical and simulation results}

The analytical and simulation SEPs under perfect CSI and imperfect CSI are shown in Fig. 1 and Fig. 2, respectively. Note that for the situation with perfect CSI, $c_{h \hat{h}}^{2}=1$. It is observed that the analytical results are very close to the simulation ones. It is demonstrated that the proposed analysis can yield accurate results. 


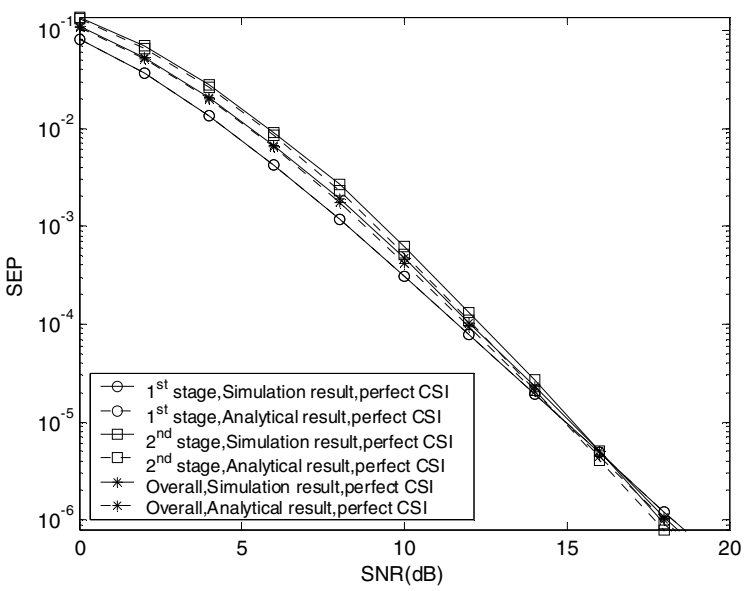

Fig. 1 Analytical and simulation SEPs under perfect CSI.

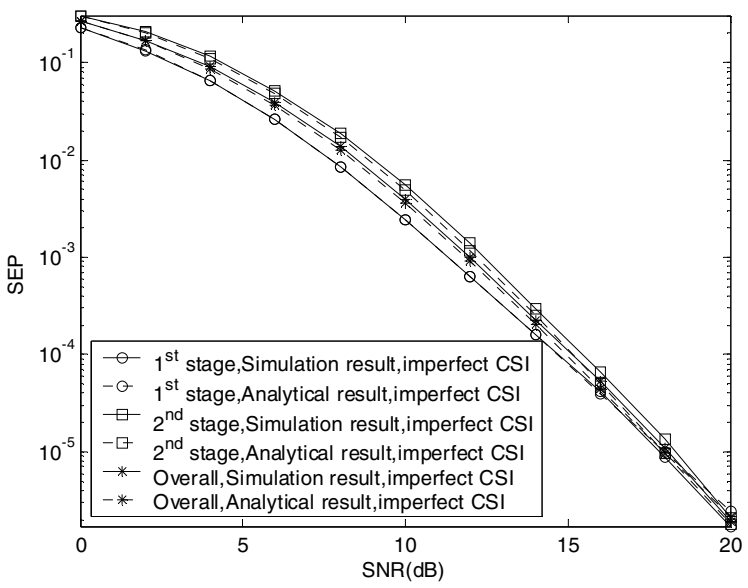

Fig. 2 Analytical and simulation SEPs under imperfect CSI.

\section{B. The effects of optimal ordering}

The effects of optimal ordering on the cumulative density functions (c.d.f) of $\omega_{2}$ and $\omega_{1}$ (recall that $\omega_{2}$ and $\omega_{1}$ are the squares of the normalized diagonal elements $r_{2,2}$ and $r_{1,1}$ respectively) are shown in Fig. 3. It is shown that by adopting the optimal ordering, the distribution of $\omega_{2}$ has been right shifted while on the contrary, the distribution of $\omega_{1}$ has been left shifted. It means that, from the statistical point of view, the optimal ordering will increase the post-detection SNR at the $1^{\text {st }}$ stage (see (11)), but decrease the post-detection SNR at the $2^{\text {nd }}$ stage (see (16) and (20)). Therefore, from (12), it is expected that the performance at the $1^{\text {st }}$ stage will be improved. However, the performance at the $2^{\text {nd }}$ stage is not such straight-forward. Equation (14) implies that, on one hand, the SEP at the $2^{\text {nd }}$ stage will be degraded because of the decrease of post-detection SNR; on the other hand, it will be improved by the mitigation of the error propagation from the $1^{\text {st }}$ stage.
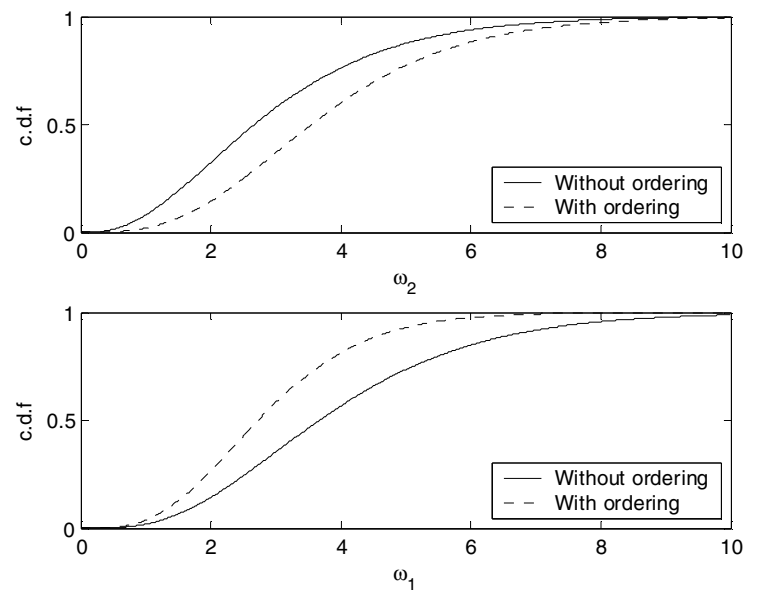

Fig. 3 The effects of ordering on the c.d.f of $\omega_{2}$ and $\omega_{1}$.

The effects of ordering on the SEP performance of the $1^{\text {st }}$ and $2^{\text {nd }}$ stages are shown in Fig. 4 and Fig. 5 respectively. And the effect of ordering on the overall SEP performance is then presented in Fig. 6. Since the analytical results are very close to the simulation ones, here only the analytical results are shown for both detections without and with ordering.

It is observed that: 1) as expected, the SEP performance at the $1^{\text {st }}$ stage is improved by optimal ordering irrespective of SNR;2) the effect of ordering on the SEP at the $2^{\text {nd }}$ stage varies with SNR. The ordering degrades the SEP performance at the $2^{\text {nd }}$ stage under low SNR, while it improves the performance when SNR is high. It is inferred that under low SNR, the decrease of the post-detection SNR dominates the effect of ordering and thus the SEP performance is degraded, whereas the mitigation of error propagation dominates under high SNR and thus the SEP performance is improved; 3) the overall SEP performance is improved by the ordering procedure. The improvement is not significant in low SNR region, but it is considerable in high SNR region.

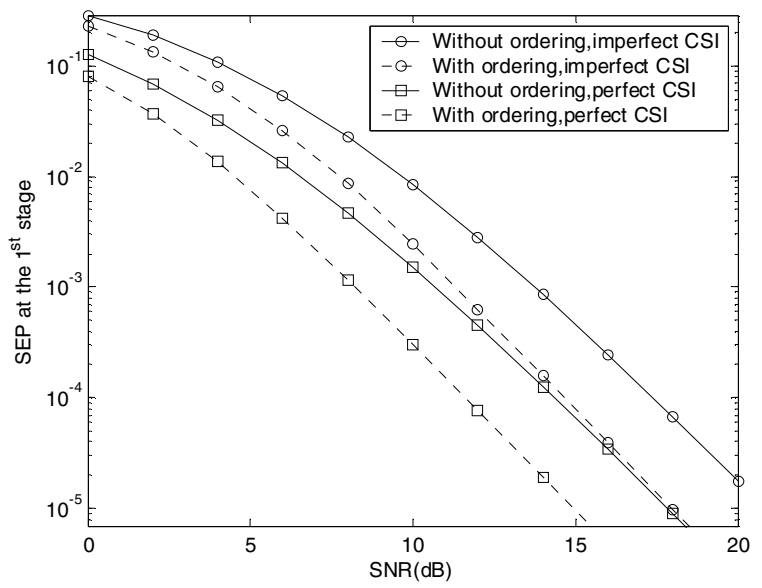

Fig. 4 Effect of ordering on the SEP performance at the $1^{\text {st }}$ stage. 


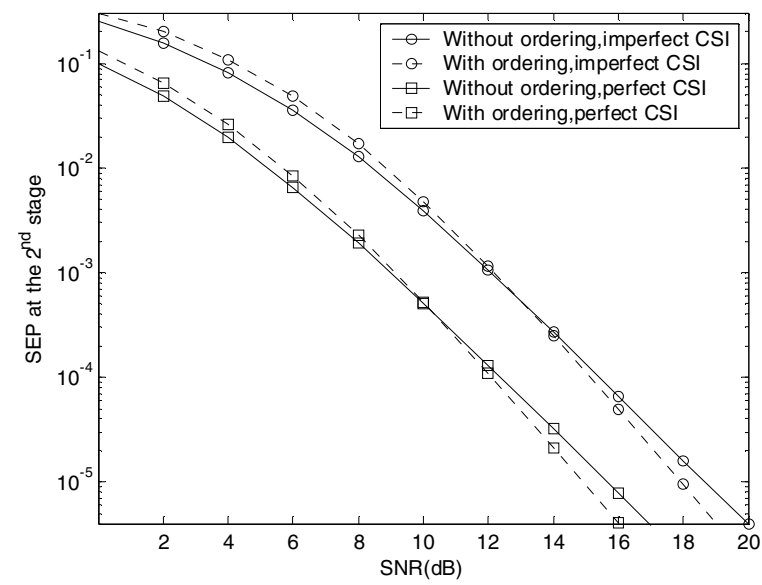

Fig. 5 Effect of ordering on the SEP performance at the $2^{\text {nd }}$ stage.

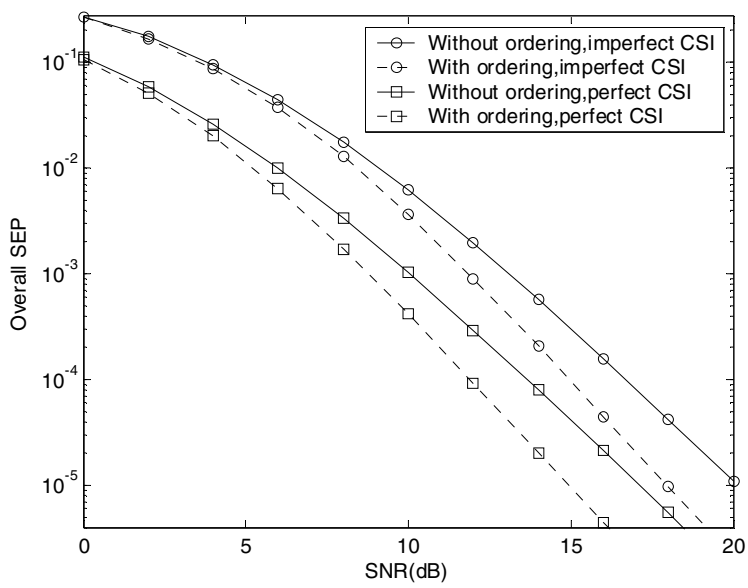

Fig. 6 Effect of ordering on the overall SEP performance.

\section{CONCLUSIONS}

This paper has presented the SEP analysis of the V-BLAST detection with optimal ordering for systems with two transmitted antennas. The post-detection SNR at each detection stage has been derived and the SEPs of the two stages have been given in closed-form. The paper has modeled the effect of imperfect channel estimation as well as the effect of error propagation whereas the existing methods have difficulty in obtaining a solution. Monte-Carlo simulations have demonstrated that the proposed analysis yield accurate results.

\section{ACKNOWLEDGEMENT}

This work was supported by the Hong Kong research grants council (Grant No.: 7160/06E) and also partly supported by the Global COE program of the Tohoku University.

\section{REFERENCES}

[1] P.W. Wolniansky, G.J. Foschini, G.D. Golden and R.A. Valenzuela, "VBLAST: an Architecture for Realizing very High Data Rates over The Rich Scattering Wireless Channel," Proceeding of URSI International Symposium on Signals, Systems, and Electronics, pp. 295-300, 1998.

[2] S. Loyka and F. Gagnon, "Performance Analysis of the V-BLAST Algorithm: An Analytical Approach," IEEE Transactions on Wireless Communications, vol. 3, pp. 1326-1337, July 2004

[3] R. T. Xu and F.C.M. Au, "Analytical Approach of V-BLAST Performance with Two Transmit Antennas," IEEE Conference on Wireless Communications and Networking, vol. 1, pp.396 - 401, March 2005.

[4] S. Loyka and F. Gagnon, "V-BLAST without Optimal Ordering: Analytical Performance Evaluation for Rayleigh Fading Channels," IEEE Transactions on Wireless Communications, vol. 54, pp. 1109-1120, June 2006.

[5] C. Shen, Y. Zhu, S. D. Zhou and J. J. Jiang, "On the Performance of VBLAST with Zero-forcing Successive Interference Cancellation Receiver," IEEE Globecom 2004, vol. 5, pp. 2818 - 2822, November 2004.

[6] R. Narasimhan, "Error Propagation Analysis of V-BLAST with ChannelEstimation Errors, IEEE Transactions on Communications," vol. 53, pp. 27-31, January 2005.

[7] W. Y. Li and N. C. Beaulieu, "Effects of Channel-Estimation Errors on Receiver Selection-combining Schemes for Alamouti MIMO Systems with BPSK," IEEE Transactions on Communications, vol. 54, pp. 169-178, January 2006.

[8] S. J. Grant and J. K. Cavers, "Performance Enhancement through Joint Detection of Co-channel Signals Using Diversity Arrays," IEEE Transactions on Communications, vol. 46, pp. 1038-1049, August 1998.

[9] J.G. Proakis, Digital Communications, McGraw Hill, 4th edition, 2001.

[10] C. Shen, H. R. Zhuang, L. Dai and S. D. Zhou, "Detection Algorithm Improving V-BLAST Performance over Error Propagation," IEE Electronics Letters, vol. 39, pp.1007 - 1008, June 2003.

[11] V. V. Prasolov, "Problems and Theorems in Linear Algebra," American Mathematical Society, 1994.

[12] T. W. Anderson, An Introduction to Multivariate Analysis, 3rd edition, New York: Wiley, 2003.

[13] M. Kay Steven, Fundamentals of Statistical Signal Processing, Prentice Hall, 1993. 